\title{
MAXIMA OF STOCHASTIC PROCESSES DRIVEN BY FRACTIONAL BROWNIAN MOTION
}

\author{
BORIS BUCHMANN, ${ }^{*}$ Australian National University \\ CLAUDIA KLÜPPELBERG, ${ }^{* *}$ Munich University of Technology
}

\begin{abstract}
We study stationary processes given as solutions to stochastic differential equations driven by fractional Brownian motion. This rich class includes the fractional OrnsteinUhlenbeck process and those processes that can be obtained from it by state space transformations. An explicit formula in terms of Euler's $\Gamma$-function describes the asymptotic behaviour of the covariance function of the fractional Ornstein-Uhlenbeck process near zero, which, by an application of Berman's condition, guarantees that this process is in the maximum domain of attraction of the Gumbel distribution. Necessary and sufficient conditions on the state space transforms are stated to classify the maximum domain of attraction of solutions to stochastic differential equations driven by fractional Brownian motion.
\end{abstract}

Keywords: Extreme-value theory; fractional Brownian motion; fractional OrnsteinUhlenbeck process; fractional stochastic differential equation; long-range dependence; partial maximum; maximum domain of attraction; state space transform

2000 Mathematics Subject Classification: Primary 60G70; 60G15

Secondary 60G10; 60H20

\section{Introduction}

Let $(\Omega, \mathcal{F}, \mathrm{P})$ be a complete probability space carrying a two-sided fractional Brownian motion $\left(B_{t}^{H}\right)_{t \in \mathbb{R}}$ with Hurst index $H \in(0,1)$, i.e. a centred Gaussian process with covariance function

$$
\mathrm{E}\left(B_{t}^{H} B_{s}^{H}\right)=\frac{1}{2}\left(|t|^{2 H}+|s|^{2 H}-|t-s|^{2 H}\right), \quad s, t \in \mathbb{R} .
$$

Fractional Brownian motion has stationary increments and is self-similar, i.e. for all $c \in \mathbb{R}$,

$$
\left(B_{c t}^{H}\right) \stackrel{\mathrm{D}}{=}|c|^{H}\left(B_{t}^{H}\right), \quad t \in \mathbb{R}
$$

where ' $\stackrel{\text { D, }}{=}$ denotes equality in distribution; in particular, $B_{0}^{H}=0$. A Hurst index of $H=\frac{1}{2}$ corresponds to standard Brownian motion. Further properties can be found in [14].

Our goal is to investigate the asymptotic behaviour of partial maxima of stationary solutions $X$ given by a stochastic differential equation of the form

$$
X_{t}-X_{s}=\int_{s}^{t} \mu\left(X_{u}\right) \mathrm{d} u+\int_{s}^{t} \sigma\left(X_{u}\right) \mathrm{d} B_{u}^{H}, \quad s \leq t,
$$

\footnotetext{
Received 19 January 2005; revision received 23 March 2005.

* Postal address: Centre of Excellence for Mathematics and Statistics of Complex Systems, Mathematical Science Institute, Australian National University, Canberra, ACT 0200, Australia.

Email address: boris.buchmann@maths.anu.edu.au

** Postal address: Centre for Mathematical Sciences, Munich University of Technology, D-85747 Garching, Germany. Email address: cklu@ma.tum.de
} 
for continuous functions $\mu$ and $\sigma>0$. The integrals are interpreted pathwise as RiemannStieltjes integrals. For an analytic treatment and conditions on $\mu$ and $\sigma$ for the existence of such solutions, we refer the reader to [5].

A prominent example is the Ornstein-Uhlenbeck model, which corresponds to linear $\mu$ and constant $\sigma$. More precisely, for $\gamma, \sigma>0$, we define the fractional Ornstein-Uhlenbeck process (FOUP) by

$$
O_{t}^{H, \gamma, \sigma}=\sigma \int_{-\infty}^{t} \mathrm{e}^{-\gamma(t-s)} \mathrm{d} B_{s}^{H}, \quad t \in \mathbb{R} .
$$

The process $O^{H, \gamma, \sigma}=\left(O_{t}^{H, \gamma, \sigma}\right)_{t \in \mathbb{R}}$ is stationary and provides a pathwise solution to the stochastic differential equation

$$
O_{t}-O_{s}=-\gamma \int_{s}^{t} O_{u} \mathrm{~d} u+\sigma\left(B_{t}^{H}-B_{s}^{H}\right), \quad s \leq t .
$$

As $O^{H, \gamma, \sigma}$ is a Gaussian process, classical results due to Pickands [11] and Berman [1] apply, giving a limit result for partial maxima. Standard references summarizing the extreme-value theory of Gaussian processes are [2], [10], and [12]. We present explicit calculations concerning the FOUP in Section 2.

As was shown in [5], under certain conditions on $\mu$ and $\sigma$, the solution $X$ to (1.2) can be represented as a state space transform of the FOUP. Consequently, in Section 3, we investigate the full class of processes that can be obtained from the FOUP by state space transforms. We give condition necessary and sufficient to characterize the maximum domain of attraction for such processes.

In Section 4, we return to the original problem. Working within the framework of [5], we obtain conditions necessary and sufficient to characterize the maximum domain of attraction for stationary solutions to (1.2). These results are based on asymptotic inversion results, whose proofs can be found in Appendix C.

Our approach bears some similarity to those of Davis [8] and Borkovec and Klüppelberg [4], who investigated the extremal behaviour of diffusion processes given as solutions to stochastic differential equations driven by Brownian motion. Whereas they used the classical OrnsteinUhlenbeck process as a reference process to obtain the extreme behaviour of other families of diffusion processes, we use the FOUP instead. In those papers, scale functions and time changes of the classical Ornstein-Uhlenbeck process are the core arguments. Since such methods do not exist for processes driven by fractional Brownian motion, we use a slightly different, but related, approach.

\section{Maxima of fractional Ornstein-Uhlenbeck processes}

For any continuous-time process $X=\left(X_{t}\right)_{t \geq 0}$, we say that it belongs to the domain of attraction of some extreme-value distribution $G$, and we write $X \in \operatorname{MDA}(G)$, if there exist normalizing constants $a_{T}>0$ and $b_{T} \in \mathbb{R}$, for $T \geq 0$, such that

$$
a_{T}^{-1}\left(\max _{0 \leq t \leq T} X_{t}-b_{T}\right) \stackrel{\mathrm{D}}{\rightarrow} G
$$

where, throughout, ' $\stackrel{\mathrm{D}}{\rightarrow}$ ' denotes convergence in distribution as $T \rightarrow \infty$.

Possible extreme-value distributions are the Fréchet distribution $\Phi_{\alpha}, \alpha>0$, the Gumbel distribution $\Lambda$, and the Weibull distribution $\Psi_{\alpha}, \alpha>0$. For details on standard extreme-value theory, we refer the reader to [9] or [10]. 
In this section, we derive the extreme behaviour of the FOUP given in (1.4). As it is a Gaussian process, we can apply the theory of [1], [2], and [11]. The behaviour of partial maxima of a Gaussian process can be related to the behaviour of the covariance function at 0 and infinity. For any $t \in \mathbb{R}$, we define the covariance function

$$
\rho_{H, \gamma, \sigma}(h)=\mathrm{E}\left(O_{t}^{H, \gamma, \sigma} O_{t+h}^{H, \gamma, \sigma}\right), \quad h \in \mathbb{R} .
$$

As the FOUP is stationary, the function $\rho_{H, \gamma, \sigma}(\cdot)$ does not depend on $t$. Throughout the paper, we write $O^{H} \equiv O^{H, 1,1}$ and $\rho_{H} \equiv \rho_{H, 1,1}$. In the following lemma, we summarize some properties of $\rho$ (see Appendix A for a proof).

Lemma 2.1. (a) Symmetry: $\rho_{H, \gamma, \sigma}(h)=\rho_{H, \gamma, \sigma}(|h|)$.

(b) Scaling property: $\rho_{H, \gamma, \sigma}(h)=\left(\sigma^{2} / \gamma^{2 H}\right) \rho_{H}(\gamma h)$.

(c) Asymptotic behaviour at infinity [7]:

$$
\rho_{H, \gamma, \sigma}(h)= \begin{cases}\frac{1}{2} \frac{\sigma^{2}}{\gamma} \mathrm{e}^{-\gamma|h|} & \text { for } h \in \mathbb{R}, \text { if } H=\frac{1}{2}, \\ O\left(h^{2 H-2}\right) & \text { for } h \rightarrow \infty, \text { if } H \neq \frac{1}{2} .\end{cases}
$$

(d) Asymptotic behaviour for $h \rightarrow 0$ :

$$
\rho_{H, \gamma, \sigma}(h)= \begin{cases}\frac{\Gamma(2 H+1)}{2} \frac{\sigma^{2}}{\gamma^{2 H}}-\frac{\sigma^{2}}{2}|h|^{2 H}+o(|h|), & H<\frac{1}{2}, \\ \frac{1}{2} \frac{\sigma^{2}}{\gamma} \mathrm{e}^{-\gamma|h|}, & H=\frac{1}{2}, \\ \frac{\Gamma(2 H+1)}{2} \frac{\sigma^{2}}{\gamma^{2 H}}-\frac{\sigma^{2}}{2}|h|^{2 H}+\frac{\Gamma(2 H+1)}{4} \frac{\sigma^{2}}{\gamma^{2 H-2}}|h|^{2}+o\left(|h|^{2}\right), & H>\frac{1}{2} .\end{cases}
$$

We can now formulate a result for the partial maxima of a FOUP.

Theorem 2.1. Let $\gamma, \sigma>0$. Then

$$
\left(\sigma a_{T}^{H, \gamma}\right)^{-1}\left(\max _{0 \leq t \leq T} O_{t}^{H, \gamma, \sigma}-\sigma b_{T}^{H, \gamma}\right) \stackrel{\mathrm{D}}{\rightarrow} \Lambda,
$$

where

$$
\begin{aligned}
a_{T}^{H, \gamma} & =\gamma^{-H} \frac{\Gamma(2 H+1)^{1 / 2}}{2(\log T)^{1 / 2}}, \\
b_{T}^{H, \gamma} & =\gamma^{-H} \frac{\Gamma(2 H+1)^{1 / 2}}{2^{1 / 2}}\left(2(\log T)^{1 / 2}+\frac{1-H}{2 H} \frac{\log \log T}{(\log T)^{1 / 2}}+\frac{\mathcal{C}(H, \gamma)}{(\log T)^{1 / 2}}\right), \\
\mathcal{C}(H, \gamma) & =\log \left(\gamma \Gamma(2 H+1)^{-1 / 2 H} \mathscr{H}_{2 H}(2 \pi)^{-1 / 2} 2^{(1-H) / 2 H}\right),
\end{aligned}
$$

and $\mathscr{H}_{2 H}$ is Pickands' number, a constant.

Proof. We apply the following result on Gaussian processes, due to Pickands [11] and Berman [1]; see, e.g. [10, Theorem 12.3.5]. For any normal process $\left(X_{t}\right)_{t \geq 0}$ such that Berman's conditions hold, i.e.

$$
\mathrm{E}\left(X_{h} X_{0}\right)= \begin{cases}1-d|h|^{2 H}+o\left(|h|^{2 H}\right), & h \rightarrow 0, \\ o\left((\log h)^{-1}\right), & h \rightarrow \infty\end{cases}
$$


for constants $d>0$ and $H \in(0,1)$, we have

$$
(2 \log T)^{1 / 2}\left(\max _{0 \leq t \leq T} X_{t}-\beta_{T}(H, d)\right) \stackrel{\mathrm{D}}{\rightarrow} \Lambda,
$$

where

$$
\begin{aligned}
\beta_{T}(H, d) & =(2 \log T)^{1 / 2}+\frac{1-H}{2 H} \frac{\log \log T}{(2 \log T)^{1 / 2}}+\frac{\psi(H, d)}{(2 \log T)^{1 / 2}}, \\
\psi(H, d) & =\log \left(d^{1 / 2 H} \mathscr{H}_{2 H}(2 \pi)^{-1 / 2} 2^{(1-H) / 2 H}\right) .
\end{aligned}
$$

For $t \in \mathbb{R}$, define a normal process $X_{t}^{H, \gamma, \sigma}:=\left(\rho_{H, \gamma, \sigma}(0)\right)^{-1 / 2} O_{t}^{H, \gamma, \sigma}$. Condition (2.2) for $h \rightarrow \infty$ is ensured by (2.1). From Lemma 2.1(d) we obtain, for $d=\gamma^{2 H} / \Gamma(2 H+1)$,

$$
\mathrm{E}\left(X_{h}^{H, \gamma, \sigma} X_{0}^{H, \gamma, \sigma}\right)=1-\frac{1}{2}\left(\rho_{H, \gamma, \sigma}(0)\right)^{-1} \sigma^{2}|h|^{2 H}+o\left(|h|^{2 H}\right)=1-d|h|^{2 H}+o\left(|h|^{2 H}\right) .
$$

Hence, for this value of $d$, we have

$$
(2 \log T)^{1 / 2}\left(\max _{0 \leq t \leq T} X_{t}^{H, \gamma, \sigma}-\beta_{T}(H, d)\right) \stackrel{\mathrm{D}}{\rightarrow} \Lambda
$$

and, therefore, with $a_{T}^{H, \gamma}$ as defined in the theorem, we obtain

$$
\left(\sigma a_{T}^{H, \gamma}\right)^{-1}\left(\max _{0 \leq t \leq T} O_{t}^{H, \gamma, \sigma}-\frac{\sigma}{\gamma^{H}}\left(\frac{\Gamma(2 H+1)}{2}\right)^{1 / 2} \beta_{T}(H, d)\right) \stackrel{\mathrm{D}}{\rightarrow} \Lambda .
$$

Choosing $\mathcal{C}(H, \gamma)=\psi\left(H, \gamma^{2 H} / \Gamma(2 H+1)\right)$ proves the result.

Remark 2.1. (a) Henceforth, we write $O_{t}^{H, \gamma} \equiv O_{t}^{H, \gamma, 1}$. Setting $M_{T}^{H, \gamma}=\max _{0 \leq t \leq T} O_{t}^{H, \gamma}$, we see that

$$
\frac{b_{T}^{H, \gamma}}{a_{T}^{H, \gamma}}\left(\frac{M_{T}^{H, \gamma}}{b_{T}^{H, \gamma}}-1\right) \stackrel{\mathrm{D}}{\rightarrow} \Lambda .
$$

As $b_{T}^{H, \gamma} / a_{T}^{H, \gamma} \rightarrow \infty$ we conclude that $M_{T}^{H, \gamma} / b_{T}^{H, \gamma} \stackrel{\mathrm{P}}{\rightarrow} 1$, where $\stackrel{\text { P }}{\rightarrow}$ ' denotes convergence in probability. The distribution of $M_{T}^{H, \gamma}$ thus becomes less spread around $b_{T}^{H, \gamma}$ as $T$ becomes large. Consequently, $b_{T}^{H, \gamma}$ quite precisely describes the growth of the partial maxima for large $T$.

(b) Observe that $a_{T}^{H, \gamma} b_{T}^{H, \gamma} \rightarrow 1 / \delta^{H, \gamma}=\Gamma(2 H+1) /\left(\gamma^{2 H} 2^{1 / 2}\right)$. The convergence-to-types theorem (see [9, Theorem A1.5]) allows for different scaling, namely

$$
\delta^{H, \gamma} b_{T}^{H, \gamma}\left(M_{T}^{H, \gamma}-b_{T}^{H, \gamma}\right) \stackrel{\mathrm{D}}{\rightarrow} \Lambda .
$$

(c) For the definition of Pickands' number, we refer the reader to [10]. The precise shape of the curve $H \mapsto \mathscr{H}_{2 H}$ is unknown, but a simulated curve can be found in [6].

\section{State space transforms and extremes}

In this section, we extend the result on the maximum domain of attraction for the FOUP to more general processes. We will use the notation of Remark 2.1 throughout; in particular, we set $\sigma=1$. 
In [5], the authors termed a function $f: \mathbb{R} \rightarrow \mathbb{R}$ a state space transform (SST) if $f$ is continuous and strictly increasing. An SST $f$ maps $\mathbb{R}$ to an open interval $I=(l, r)=f(\mathbb{R})$ called the state space of $f$. If we define $X_{t}^{H, \gamma, f}:=f\left(O_{t}^{H, \gamma}\right), t \in \mathbb{R}$, this yields a rich class of stationary processes driven by fractional Brownian motion on arbitrary open intervals $I$.

The next theorem gives necessary and sufficient conditions on the SST $f$ to have $X^{H, \gamma, f} \in$ $\operatorname{MDA}(\Lambda)$.

Theorem 3.1. Let $f: \mathbb{R} \rightarrow \mathbb{R}$ be an SST with $X_{t}^{H, \gamma, f}:=f\left(O_{t}^{H, \gamma}\right), t \in \mathbb{R}$, as above.

(a) Assume that

$$
\lim _{y \rightarrow \infty} \frac{f(y+x / y)-f(y)}{f(y+1 / y)-f(y)}=x \quad \text { for all } x \in \mathbb{R} .
$$

Then, for $\delta^{H, \gamma}$ as in Remark 2.1(b), we have

$$
\frac{\delta^{H, \gamma}}{f\left(b_{T}^{H, \gamma}+1 / b_{T}^{H, \gamma}\right)-f\left(b_{T}^{H, \gamma}\right)}\left(\max _{0 \leq t \leq T} X_{t}^{H, \gamma, f}-f\left(b_{T}^{H, \gamma}\right)\right) \stackrel{\mathrm{D}}{\rightarrow} \Lambda .
$$

(b) Assume that there exist normalizing constants $\tilde{a}_{T}>0$ and $\tilde{b}_{T} \in \mathbb{R}$ such that

$$
\tilde{a}_{T}^{-1}\left(\max _{0 \leq t \leq T} X_{t}^{H, \gamma, f}-\tilde{b}_{T}\right) \stackrel{\mathrm{D}}{\rightarrow} \Lambda .
$$

Then (3.1) holds and possible choices of the normalizing constants are

$$
\tilde{a}_{T}=\frac{1}{\delta^{H, \gamma}}\left(f\left(b_{T}^{H, \gamma}+\frac{1}{b_{T}^{H, \gamma}}\right)-f\left(b_{T}^{H, \gamma}\right)\right), \quad \tilde{b}_{T}=f\left(b_{T}^{H, \gamma}\right) .
$$

Proof. Let $M_{T} \equiv M_{T}^{H, \gamma}$ and $\tilde{M}_{T}=\max _{0 \leq t \leq T} X_{t}^{H, \gamma, f}$. As $f$ is increasing, $\tilde{M}_{T}=f\left(M_{T}\right)$. We write $b_{T} \equiv b_{T}^{H, \gamma}$ and $\delta \equiv \delta^{H, \gamma}$ and recall that $b_{T} \rightarrow \infty$ for $T \rightarrow \infty$. Furthermore, observe that $T \mapsto b_{T}$ is strictly increasing for all sufficiently large $T$.

(a) For such $T$, the function $g_{T}: \mathbb{R} \rightarrow \mathbb{R}$,

$$
g_{T}(x)=\delta \frac{f\left(b_{T}+x /\left(\delta b_{T}\right)\right)-f\left(b_{T}\right)}{f\left(b_{T}+1 / b_{T}\right)-f\left(b_{T}\right)},
$$

is well defined. Assumption (3.1) implies that $\lim _{T \rightarrow \infty} g_{T}(x)=x$ for all $x \in \mathbb{R}$. Furthermore,

$$
\begin{aligned}
\mathrm{P}\left(M_{T} \leq b_{T}+\frac{x}{\delta b_{T}}\right) & =\mathrm{P}\left(\frac{\delta}{f\left(b_{T}+1 / b_{T}\right)-f\left(b_{T}\right)}\left(f\left(M_{T}\right)-f\left(b_{T}\right)\right) \leq g_{T}(x)\right) \\
& =\mathrm{P}\left(\frac{\delta}{f\left(b_{T}+1 / b_{T}\right)-f\left(b_{T}\right)}\left(\tilde{M}_{T}-f\left(b_{T}\right)\right) \leq g_{T}(x)\right) .
\end{aligned}
$$

In particular, by Remark 2.1(b), the left-hand side converges pointwise to $\Lambda(x)$. Thus, Lemma B.1(a) (of Appendix B) applies.

(b) As above, we write $\mathrm{P}\left(M_{T} \leq b_{T}+x /\left(\delta b_{T}\right)\right)=\mathrm{P}\left(\tilde{a}_{T}^{-1}\left(\tilde{M}_{T}-\tilde{b}_{T}\right) \leq \tilde{g}_{T}(x)\right)$, where

$$
\tilde{g}_{T}(x)=\tilde{a}_{T}^{-1}\left(f\left(b_{T}+\frac{x}{\delta b_{T}}\right)-\tilde{b}_{T}\right) .
$$


By Lemma B.1(b), we find that $\tilde{g}_{T}(x) \rightarrow x$ for all $x \in \mathbb{R}$. In particular,

$$
\begin{aligned}
\frac{f\left(b_{T}\right)-\tilde{b}_{T}}{\tilde{a}_{T}} & =\tilde{g}_{T}(0) \rightarrow 0, \\
\frac{1}{\delta} \frac{f\left(b_{T}+1 / b_{T}\right)-f\left(b_{T}\right)}{\tilde{a}_{T}} & =\frac{1}{\delta}\left(\tilde{g}_{T}(\delta)-g_{T}(0)\right) \rightarrow 1 .
\end{aligned}
$$

By the convergence-to-types theorem, we conclude that $\left(f\left(b_{T}+1 / b_{T}\right)-f\left(b_{T}\right)\right) / \delta$ and $f\left(b_{T}\right)$ are possible choices for $\tilde{a}_{T}$ and $\tilde{b}_{T}$, respectively. Substituting $\tilde{a}_{T}=\left(f\left(b_{T}+1 / b_{T}\right)-f\left(b_{T}\right)\right) / \delta$ and $\tilde{b}_{T}=f\left(b_{T}\right)$ into (3.2) yields

$$
\left(f\left(b_{T}+\frac{x}{b_{T}}\right)-f\left(b_{T}\right)\right)\left(f\left(b_{T}+\frac{1}{b_{T}}\right)-f\left(b_{T}\right)\right)^{-1}=\frac{1}{\delta} \tilde{g}_{T}(\delta x),
$$

and the right-hand side converges to $x$ for all $x \in \mathbb{R}$; thus, (3.1) holds.

The following example illustrates (3.1).

Example 3.1. Let $q \in(0,2]$ and let $f: \mathbb{R} \rightarrow \mathbb{R}$ be an SST given by $f(x)=\mathrm{e}^{x^{q}}, x>0$.

(a) If $q \in(0,2)$ then, for all $x \in \mathbb{R}$, we have

$$
\lim _{y \rightarrow \infty} \frac{f(y+x / y)-f(y)}{f(y+1 / y)-f(y)}=\lim _{y \rightarrow \infty} \frac{\exp \left(y^{q}\left[\left(1+x / y^{2}\right)^{q}-1\right]\right)-1}{\exp \left(y^{q}\left[\left(1+1 / y^{2}\right)^{q}-1\right]\right)-1}=x .
$$

Therefore, $X^{H, \gamma, f} \in \operatorname{MDA}(\Lambda)$.

(b) If $q=2$ then, for all $x \in \mathbb{R}$, we have

$$
\lim _{y \rightarrow \infty} \frac{f(y+x / y)-f(y)}{f(y+1 / y)-f(y)}=\lim _{y \rightarrow \infty} \frac{\mathrm{e}^{2 x+x^{2} / y^{2}}-1}{\mathrm{e}^{2+1 / y^{2}}-1}=\frac{\mathrm{e}^{2 x}-1}{\mathrm{e}^{2}-1} .
$$

Thus, $X^{H, \gamma, f} \notin \operatorname{MDA}(\Lambda)$. In fact, Theorem 3.2, below, will show that $X^{H, \gamma, f} \in \operatorname{MDA}\left(\Phi_{\alpha}\right)$.

Under the additional hypothesis of differentiability, the next corollary provides an efficient method to calculate normalizing constants. This is then illustrated in Corollaries 3.2 and 3.3.

Corollary 3.1. Let $f$ be an SST differentiable on $\left(z_{0}, \infty\right)$, with $f^{\prime}(z)>0$ for all $z \in\left(z_{0}, \infty\right)$. Assume that

$$
\lim _{z \rightarrow \infty} \frac{f^{\prime}(z+x / z)}{f^{\prime}(z)}=1 \text { locally uniformly in } x .
$$

Then

$$
\frac{1}{a_{T}^{H, \gamma} f^{\prime}\left(b_{T}^{H, \gamma}\right)}\left(\max _{0 \leq t \leq T} X_{t}^{H, \gamma, f}-f\left(b_{T}^{H, \gamma}\right)\right) \stackrel{\mathrm{D}}{\rightarrow} \Lambda .
$$

Proof. Let $x \in \mathbb{R}$. For all sufficiently large $y>0$, we can find $\theta_{y} \in[0,1]$ and $\tilde{\theta}_{y} \in[0,1]$ such that

$$
\frac{f(y+x / y)-f(y)}{f(y+1 / y)-f(y)}=x \frac{f^{\prime}\left(y+\theta_{y} x / y\right)}{f^{\prime}(y)} \frac{f^{\prime}(y)}{f^{\prime}\left(y+\tilde{\theta}_{y} / y\right)} \rightarrow x, \quad y \rightarrow \infty .
$$

Therefore, (3.1) follows from (3.3) and, consequently, $X^{H, \gamma, f} \in \operatorname{MDA}(\Lambda)$. 
Furthermore, for some $\bar{\theta}_{T} \in[0,1]$ and the quantity $\delta^{H, \gamma}$ in Remark 2.1(b), we have

$$
\frac{1}{\delta^{H, \gamma}} \frac{f\left(b_{T}^{H, \gamma}+1 / b_{T}^{H, \gamma}\right)-f\left(b_{T}^{H, \gamma}\right)}{a_{T}^{H, \gamma} f^{\prime}\left(b_{T}^{H, \gamma}\right)}=\frac{1}{\delta^{H, \gamma}} \frac{1}{a_{T}^{H, \gamma} b_{T}^{H, \gamma}} \frac{f^{\prime}\left(b_{T}^{H, \gamma}+\bar{\theta}_{T} / b_{T}^{H, \gamma}\right)}{f^{\prime}\left(b_{T}^{H, \gamma}\right)} \rightarrow 1 ;
$$

thus, (3.4) follows by the convergence-to-types theorem.

Corollary 3.2. Let $\ell$ be a slowly varying function on $\left[x_{0}, \infty\right)$ for some $x_{0}>0$, i.e. $\ell:\left[x_{0}, \infty\right)$ $\rightarrow \mathbb{R}^{+}$is measurable and $\lim _{x \rightarrow \infty} \ell(t x) / \ell(x)=1$ for all $t>0$. If $f$ is an SST with state space $I=(l, r)$, differentiable on $\left(x_{0}, \infty\right)$ and such that, for some $p \in \mathbb{R}$,

$$
f^{\prime}(x)=x^{p} \ell(x) \text { for all } x>x_{0},
$$

then $X^{H, \gamma, f} \in \operatorname{MDA}(\Lambda)$.

Define

$$
\begin{aligned}
c_{p}^{H, \gamma} & =2^{(p-2) / 2} \gamma^{-H(p+1)} \Gamma(2 H+1)^{(p+1) / 2}, \\
\tilde{a}_{T} & =c_{p}^{H, \gamma}(\log T)^{(p-1) / 2} \ell\left((\log T)^{1 / 2}\right) .
\end{aligned}
$$

Then $\tilde{a}_{T}$ and $\tilde{b}_{T}=f\left(b_{T}^{H, \gamma}\right)$ are a possible choice of normalizing constants.

Proof. By [3, Theorem 1.5.2], convergence in regular variation is locally uniform; thus, locally uniformly in $x$,

$$
\lim _{z \rightarrow \infty} \frac{f^{\prime}(z+x / z)}{f^{\prime}(z)}=\lim _{z \rightarrow \infty} \frac{\ell\left(z\left(1+x / z^{2}\right)\right)}{\ell(z)}=1 .
$$

Consequently, $X^{H, \gamma, h} \in \operatorname{MDA}(\Lambda)$ by Corollary 3.1 .

According to (3.4), we find that $a_{T}^{H, \gamma} f^{\prime}\left(b_{T}^{H, \gamma}\right) \sim \tilde{a}_{T}$, as given in (3.5), and, thus, that $\tilde{a}_{T}$ and $\tilde{b}_{T}=f\left(b_{T}^{H, \gamma}\right)$ are a possible choice of normalizing constants, by the convergence-to-types theorem.

Corollary 3.3. Let $\ell$ be a slowly varying function on $\left[x_{0}, \infty\right)$ for some $x_{0}>0$. If $f$ is an SST with state space $I=(l, r)$, differentiable on $\left(x_{0}, \infty\right)$ and such that, for some $p \in \mathbb{R}, q \in(0,2)$, and $\kappa \neq 0$, we have

$$
f^{\prime}(x)=x^{p} \ell(x) \mathrm{e}^{\kappa x^{q}} \quad \text { for all } x>x_{0},
$$

then $X^{H, \gamma, f} \in \operatorname{MDA}(\Lambda)$.

Let $c_{p}^{H, \gamma}$ be as defined in (3.5), and define

$$
\begin{aligned}
\tilde{c}_{q}^{H, \gamma} & =2^{q / 2} \gamma^{-q H} \Gamma(2 H+1)^{q / 2}, \\
\tilde{a}_{T} & =c_{p}^{H, \gamma}(\log T)^{(p-1) / 2} \ell\left((\log T)^{1 / 2}\right) \exp \left(\kappa \tilde{c}_{q}^{H, \gamma}(\log T)^{q / 2}\right) .
\end{aligned}
$$

(a) If $\kappa>0$ then $r=\infty$ and $\tilde{a}_{T}$ and $\tilde{b}_{T}$ are a possible choice for the normalizing constants, where

$$
\tilde{b}_{T}=(q \kappa)^{-1}\left(b_{T}^{H, \gamma}\right)^{p-q+1} \ell\left(b_{T}^{H, \gamma}\right) \exp \left(\kappa\left(b_{T}^{H, \gamma}\right)^{q}\right) .
$$

(b) If $\kappa<0$ then $r<\infty$ and $\tilde{a}_{T}$ and $\tilde{b}_{T}$ are a possible choice for the normalizing constants, where

$$
\tilde{b}_{T}=r+(q \kappa)^{-1}\left(b_{T}^{H, \gamma}\right)^{p-q+1} \ell\left(b_{T}^{H, \gamma}\right) \exp \left(\kappa\left(b_{T}^{H, \gamma}\right)^{q}\right) .
$$


Proof. In view of Corollary 3.2, in order to prove (3.3) it suffices that, for $0<q<2$,

$$
(z+x / z)^{q}-z^{q}=q x z^{q-2}+o\left(z^{q-2}\right)=o(1), \quad z \rightarrow \infty,
$$

locally uniformly in $x$. Hence, Corollary 3.1 applies and $X^{H, \gamma, f} \in \operatorname{MDA}(\Lambda)$. As $q<2$, observe that

$$
a_{T}^{H, \gamma} \frac{f^{\prime}\left(b_{T}^{H, \gamma}\right)}{\tilde{a}_{T}} \sim \exp \left(O\left((\log T)^{(q-2) / 2} \log \log T\right)\right) \rightarrow 1 .
$$

If $\kappa>0$ then $f(x) \rightarrow \infty$ as $x \rightarrow \infty$ and, hence, $r=\infty$. Without loss of generality, suppose that $x_{0}>0$. For $x \geq x_{0}$, make the change of variable $z=(\log \bar{z})^{1 / q}$; this yields

$$
f(x)-f\left(x_{0}\right)=q^{-1} \int_{\exp \left(x_{0}^{q}\right)}^{\exp \left(x^{q}\right)} \bar{z}^{\kappa-1}(\log \bar{z})^{(p-q+1) / q} \ell\left((\log \bar{z})^{1 / q}\right) \mathrm{d} \bar{z} .
$$

Karamata's theorem [3, Theorem 1.6.1] applies for $\kappa>0$ and, for $\eta:=\mathrm{e}^{x^{q}} \rightarrow \infty$,

$$
q^{-1} \int_{\eta_{0}}^{\eta} \bar{z}^{\kappa-1}(\log \bar{z})^{(p-q+1) / q} \ell\left((\log \bar{z})^{1 / q}\right) \mathrm{d} \bar{z} \sim(q \kappa)^{-1} \eta^{\kappa}(\log \eta)^{(p-q+1) / q} \ell\left((\log \eta)^{1 / q}\right) .
$$

Thus, for $x \rightarrow \infty$, we have

$$
f(x)-f\left(x_{0}\right) \sim \psi(x), \quad \psi(x)=(q \kappa)^{-1} \ell(x) x^{p-q+1} \mathrm{e}^{\kappa x^{q}} .
$$

Note that $\tilde{a}_{T} \rightarrow \infty$ and $\tilde{a}_{T}^{-1} \psi\left(b_{T}^{H, \gamma}\right)=O\left((\log T)^{(2-q) / 2}\right)$; thus,

$$
\lim _{T \rightarrow \infty} \tilde{a}_{T}^{-1}\left(f\left(b_{T}^{H, \gamma}\right)-\psi\left(b_{T}^{H, \gamma}\right)\right)=0 .
$$

An application of the convergence-to-types theorem implies part (a).

The proof of part (b) is similar.

We now want to derive an analogue of Theorem 3.1 for the domain of attraction of the Fréchet distribution. To this end, we use the fact that, by a logarithmic transformation, for a suitable choice of normalizing constants $\hat{a}_{T}$,

$$
\hat{a}_{T}^{-1}(H, \gamma) \max _{0 \leq t \leq T} X_{t}^{H, \gamma, f} \stackrel{\mathrm{D}}{\rightarrow} \Phi_{\alpha},
$$

for some $\alpha>0$, if and only if

$$
\alpha\left(\max _{0 \leq t \leq T} \log X_{t}^{H, \gamma, f}-\log \hat{a}_{T}\right) \stackrel{\mathrm{D}}{\rightarrow} \Lambda .
$$

Using this result, we can translate Theorem 3.1 as follows.

Theorem 3.2. Let $f: \mathbb{R} \rightarrow \mathbb{R}$ be an SST.

(a) Assume that there exist $a \kappa>0$ and $a z_{0} \in \mathbb{R}$ such that, for all $z \geq z_{0}$, both $f(z)>0$ and

$$
\log f(z)=\frac{1}{2} \kappa z^{2}+h(z)
$$

where $h: \mathbb{R} \rightarrow \mathbb{R}$ satisfies

$$
\lim _{z \rightarrow \infty} h(z+x / z)-h(z)=0 \quad \text { for all } x \in \mathbb{R} .
$$


Then, for $\alpha=\delta^{H, \gamma} / \kappa\left(\right.$ with $\delta^{H, \gamma}$ as in Remark 2.1(b)),

$$
\frac{1}{f\left(b_{T}^{H, \gamma}\right)} \max _{0 \leq t \leq T} X_{t}^{H, \gamma, f} \stackrel{\mathrm{D}}{\rightarrow} \Phi_{\alpha}
$$

(b) Assume that there exist normalizing constants $\hat{a}_{T}>0$ such that

$$
\frac{1}{\hat{a}_{T}} \max _{0 \leq t \leq T} X_{t}^{H, \gamma, f} \stackrel{\mathrm{D}}{\rightarrow} \Phi_{\alpha} .
$$

Then a possible choice of $\hat{a}_{T}$ is $\hat{a}_{T}=f\left(b_{T}^{H, \gamma}\right)$. Furthermore, there exist a function $h: \mathbb{R} \rightarrow \mathbb{R}$ satisfying (3.7) and a $z_{0} \in \mathbb{R}$ such that both $f(z)>0$ and $\log f(z)=\frac{1}{2} \kappa z^{2}+h(z)$, where $\kappa=\delta^{H, \gamma} / \alpha$, hold for all $z \geq z_{0}$.

Proof. (a) As before, let $\tilde{M}_{T}=\max _{0 \leq t \leq T} X_{t}^{H, \gamma, f}$ and $M_{T} \equiv M_{T}^{H, \gamma}$, and write $b_{T} \equiv b_{T}^{H, \gamma}$ and $\delta \equiv \delta^{H, \gamma}$. Set $x=\alpha \log y$ for $y>0$. Observe that

$$
\begin{aligned}
\Phi_{\alpha}(y) & =\Lambda(x) \\
& =\lim _{T \rightarrow \infty} \mathrm{P}\left(M_{T} \leq b_{T}+\frac{x}{\delta b_{T}}\right) \\
& =\lim _{T \rightarrow \infty} \mathrm{P}\left(\frac{1}{f\left(b_{T}\right)} \tilde{M}_{T} \leq \frac{f\left(b_{T}+x /\left(\delta b_{T}\right)\right)}{f\left(b_{T}\right)}\right) \\
& =\lim _{T \rightarrow \infty} \mathrm{P}\left(\frac{1}{f\left(b_{T}\right)} \tilde{M}_{T} \leq y \theta_{T}(\alpha \log y)\right),
\end{aligned}
$$

where we have set

$$
\log \theta_{T}(x)=\frac{\kappa}{2}\left(\frac{x}{\delta b_{T}}\right)^{2}+h\left(b_{T}+\frac{x}{\delta b_{T}}\right)-h\left(b_{T}\right) .
$$

Assumption (3.7) implies that $y \theta_{T}(\alpha \log y) \rightarrow y$ for all $y>0$. Thus, Lemma B.1(a) applies to the limit in (3.8) and $g_{T}: \mathbb{R}^{+} \rightarrow \mathbb{R}$ with $g_{T}(y):=y \theta_{T}(\alpha \log y)$.

(b) Again, let $y>0$ and $x=\alpha \log y$. Replacing $f\left(b_{T}\right)$ by $\hat{a}_{T}$ in the proof of part (a), we obtain

$$
\Phi_{\alpha}(y)=\Lambda(x)=\lim _{T \rightarrow \infty} \mathrm{P}\left(\frac{1}{\hat{a}_{T}} \tilde{M}_{T} \leq \tilde{g}_{T}(y)\right),
$$

where $\tilde{g}_{T}: \mathbb{R}^{+} \rightarrow \mathbb{R}$ is defined by

$$
\tilde{g}_{T}(y)=\frac{1}{\hat{a}_{T}} f\left(b_{T}+\frac{\alpha}{\delta} \frac{\log y}{b_{T}}\right) .
$$

Lemma B.1(b) applies to $g_{T}$, i.e. $g_{T}(y) \rightarrow y$ for all $y \in \mathbb{R}^{+}$. Specializing to $y=1$, this yields $f\left(b_{T}\right) \sim \hat{a}_{T}$; thus, $f\left(b_{T}\right)$ is a possible choice for $\hat{a}_{T}$, by the convergence-to-types theorem.

Substituting $\hat{a}_{T}=f\left(b_{T}\right)$ and $\kappa=\delta / \alpha$ into (3.9) yields, for $T \rightarrow \infty$ and $y \in \mathbb{R}^{+}$,

$$
\frac{1}{f\left(b_{T}\right)} f\left(b_{T}+\frac{1}{\kappa} \frac{\log y}{b_{T}}\right) \rightarrow y .
$$


Equivalently, for $x \in \mathbb{R}$, we have

$$
\lim _{z \rightarrow \infty} \frac{f(z+x / z)}{f(z)}=\mathrm{e}^{\kappa x} .
$$

As $f\left(b_{T}\right) \sim \hat{a}_{T}$, where $\hat{a}_{T}>0$, there exists a $z_{0}$ such that $f(z)>0$ for all $z \geq z_{0}$. Set $h(z)=\log f(z)-\frac{1}{2} \kappa z^{2}$ for $z \geq z_{0}$ and $h(z)=1$ for $z<z_{0}$. Observe that

$$
h\left(z+\frac{x}{z}\right)-h(z)=\log \frac{f(z+x / z)}{f(z)}-\kappa x-\frac{1}{2} \frac{x^{2}}{z^{2}}, \quad z, z+\frac{x}{z} \geq z_{0} .
$$

Thus, $h$ is a function satisfying (3.7).

Remark 3.1. (a) The boundedness of $h$ in (3.6) does not imply (3.7). To see this, let $h(x)=$ $\sin \left(x^{2}\right)$; then

$$
h\left(z+\frac{x}{z}\right)-h(x)=2 \cos \left(z^{2}+x+\frac{x^{2}}{2 z^{2}}\right) \sin \left(x+\frac{x^{2}}{2 z^{2}}\right) .
$$

Thus, for all $x \in \mathbb{R} \backslash(\pi \mathbb{Z})$ the limit in (3.7) does not exist.

(b) Observe that Theorem 3.2 covers Example 3.1(b) with $\kappa=2$ and $h \equiv 0$ in (3.6). Furthermore, suppose that $h$ satisfies (3.7) and, in addition, $h(z) \rightarrow 0$ for $z \rightarrow \infty$. Then the scaling constants $\hat{a}_{T}$ depend on $\kappa$ and $b_{T}^{H, \gamma}$ only; i.e. we may choose

$$
\hat{a}_{T}=\exp \left(\frac{1}{2} \kappa\left(b_{T}^{H, \gamma}\right)^{2}\right) \sim f\left(b_{T}^{H, \gamma}\right) .
$$

(c) In general, knowledge of $\kappa$ alone is not sufficient to calculate the scaling constants $\hat{a}_{T}$. Therefore, observe that (3.7) holds for $h(x)=\kappa_{p} x^{p}, x>0, \kappa_{p} \neq 0$, even when $p \in[0,2)$. However, for any choice of $\hat{a}_{T}$ we must have

$$
\hat{a}_{T} \sim \exp \left(\frac{1}{2} \kappa\left(b_{T}^{H, \gamma}\right)^{2}+\kappa_{p}\left(b_{T}^{H, \gamma}\right)^{p}\right) .
$$

As $b_{T}^{H, \gamma} \rightarrow \infty$, the scaling constants $\hat{a}_{T}$ clearly depend on both $\kappa_{p}$ and $p$.

The following corollary complements Corollary 3.1.

Corollary 3.4. Let $f$ be an SST differentiable on $\left(z_{0}, \infty\right)$ for some $z_{0} \in \mathbb{R}$. Assume that $f(z)>0$ for all $z>z_{0}$ and that

$$
\lim _{z \rightarrow \infty} \frac{(\log f)^{\prime}(z+x / z)}{z}=\kappa \in(0, \infty)
$$

locally uniformly in $x$. Then, for $\alpha=\delta^{H, \gamma} / \kappa$, we have

$$
\frac{1}{f\left(b_{T}\right)} \max _{0 \leq t \leq T} X_{t}^{H, \gamma, f} \stackrel{\mathrm{D}}{\rightarrow} \Phi_{\alpha} .
$$

Proof. Set $h(z)=\log f(z)-\frac{1}{2} \kappa z^{2}$. Then $h$ is absolutely continuous on $\left[x_{0}, \infty\right)$ and we obtain

$$
h(z+x / z)-h(z)=x \int_{0}^{1} \frac{(\log f)^{\prime}(z+\alpha x / z)}{z} \mathrm{~d} \alpha-\kappa x-\frac{\kappa x^{2}}{2 z^{2}},
$$

the right-hand side of which tends to 0 for $z \rightarrow \infty$, by dominated convergence. Theorem 3.2 then applies, and the remainder of the proof follows. 
For completeness we state the analogous results for the Weibull distribution.

Theorem 3.3. Let $f: \mathbb{R} \rightarrow \mathbb{R}$ be an SST with state space $I=(l, r) \subseteq \mathbb{R}$, where $r<\infty$.

(a) Suppose that there exist $a \kappa>0$ and $a z_{0} \in \mathbb{R}$ such that both $r-f(z)>0$ and

$$
\log (r-f(z))=-\frac{1}{2} \kappa z^{2}+h(z),
$$

where $h: \mathbb{R} \rightarrow \mathbb{R}$ satisfies (3.7), hold for all $z \geq z_{0}$. Then, for $\alpha=\delta^{H, \gamma} / \kappa$, we have

$$
\frac{1}{r-f\left(b_{T}^{H, \gamma}\right)}\left(\max _{0 \leq t \leq T} X_{t}^{H, \gamma, f}-r\right) \stackrel{\mathrm{D}}{\rightarrow} \Psi_{\alpha} .
$$

(b) Assume that there exist normalizing constants $\bar{a}_{T}>0$ such that

$$
\frac{1}{\bar{a}_{T}}\left(\max _{0 \leq t \leq T} X_{t}^{H, \gamma, f}-r\right) \stackrel{\mathrm{D}}{\rightarrow} \Psi_{\alpha} .
$$

Then a possible choice of $\bar{a}_{T}$ is $\bar{a}_{T}=r-f\left(b_{T}^{H, \gamma}\right)$. Furthermore, there exist a function $h: \mathbb{R} \rightarrow \mathbb{R}$ satisfying (3.7) and a $z_{0} \in \mathbb{R}$ such that both $r-f(z)>0$ and

$$
\log (r-f(z))=-\frac{1}{2} \kappa z^{2}+h(z),
$$

where $\kappa=\delta^{H, \gamma} / \alpha$, hold for all $z \geq z_{0}$.

Proof. Set $x=-\alpha \log |z|$ for $z<0$ and $\alpha=\delta^{H, \gamma} / \kappa$. Observe that $\Psi_{\alpha}(z)=\Lambda(x)$. The result follows along the lines of the proof of Theorem 3.2.

We now collect results analogous to those of Remark 3.1 and Corollary 3.4.

Remark 3.2. (a) If $h$ satisfies (3.7) and, in addition, $h(z) \rightarrow 0$ for $z \rightarrow \infty$, then we may choose

$$
\bar{a}_{T}=\exp \left(-\frac{1}{2} \kappa\left(b_{T}^{H, \gamma}\right)^{2}\right) \sim r-f\left(b_{T}^{H, \gamma}\right) .
$$

(b) For $p \in[0,2), \kappa_{p} \neq 0$, and $h(x)=\kappa_{p} x^{p}, x>0$, we obtain

$$
\bar{a}_{T} \sim \exp \left(-\frac{1}{2} \kappa\left(b_{T}^{H, \gamma}\right)^{2}+\kappa_{p}\left(b_{T}^{H, \gamma}\right)^{p}\right) .
$$

Corollary 3.5. Let $f$ be an SST with state space $I=(l, r) \subseteq \mathbb{R}$, where $r<\infty$. Let $f$ be differentiable on $\left(z_{0}, \infty\right)$ for some $z_{0} \in \mathbb{R}$, and assume that $f(z)>0$ for all $z>z_{0}$ and that

$$
\lim _{z \rightarrow \infty} \frac{(\log (r-f))^{\prime}(z+x / z)}{z}=-\kappa \in(-\infty, 0)
$$

locally uniformly in $x$. Then, for $\alpha=\delta^{H, \gamma} / \kappa$, we have

$$
\frac{1}{r-f\left(b_{T}^{H, \gamma}\right)}\left(\max _{0 \leq t \leq T} X_{t}^{H, \gamma, f}-r\right) \stackrel{\mathrm{D}}{\rightarrow} \Psi_{\alpha} .
$$

Remark 3.3. Here, we have only considered SSTs of the FOUP. However, SSTs are of course more generally applicable to any stationary Gaussian process. 


\section{Maximum domains of attraction of solutions to fractional integral equations}

In this section, we return to the maximum domain of attraction problem for a family of processes defined as solutions to the stochastic differential equation (1.2). Therefore, let $I=$ $(l, r) \subseteq \mathbb{R}$ be an open, nonempty interval and let $\mu, \sigma: I \rightarrow \mathbb{R}$ be a pair of continuous functions, where $\sigma$ is nonnegative.

In [5], conditions on $\mu$ and $\sigma$ were obtained such that a stationary solution $X$ to (1.2) exists and is of the form $X=X^{H, \gamma, f}$, for some $\gamma>0$ and an SST $f$. These conditions were summarized into the concept of $H$-proper triples $(I, \mu, \sigma)$ (see [5, Definition 3.4]). For such triples, the ratio $\mu / \sigma$ possesses a unique, absolutely continuous extension $\psi: I \rightarrow \mathbb{R}$, which determines the SST $f$ and the so-called friction coefficient $\gamma$ according to the relations

$$
\gamma=-\sigma \psi^{\prime} \quad \text { Lebesgue almost everywhere on } I, \quad f^{-1}=-\frac{\psi}{\gamma} \text {. }
$$

The number $\xi:=f(0)$ is called the centre of the $H$-proper triple $(I, \mu, \sigma)$.

For the reader's convenience, we recall that, for $H$-proper triples, the function $z \mapsto 1 / \sigma(z)$ is necessarily locally integrable and the following formula holds for the inverse function $f^{-1}$ :

$$
f^{-1}(z)=\int_{\xi}^{z} \frac{\mathrm{d} w}{\sigma(w)}, \quad z \in I .
$$

We start with a simple example.

Example 4.1. (Fractional Vasicek model.) For $\sigma_{0}, \gamma>0$, let $\mu(x)=-\gamma(x-\xi), \xi \in \mathbb{R}$, and $\sigma(x) \equiv \sigma_{0}, x \in \mathbb{R}$. Define an $\operatorname{SST} f: \mathbb{R} \rightarrow \mathbb{R}$ by $f(x)=\xi+\sigma_{0} x$. The triple $(\mathbb{R}, \mu, \sigma)$ is $H$-proper for all $H \in(0,1)$ with friction coefficient $\gamma, \operatorname{SST} f$, and centre $\xi$. For this choice of $\mu$ and $\sigma$,observe that $X=X^{H, \gamma}, f$ is a solution to (1.2) and therefore serves as a natural extension to the fractional case of the usual Vasicek model driven by ordinary Brownian motion. It is a mean-reverting stationary Gaussian process. Theorem 2.1 implies that $X \in \operatorname{MDA}(\Lambda)$; more precisely,

$$
\left(\sigma_{0} a_{T}^{H, \gamma}\right)^{-1}\left(\max _{0 \leq t \leq T} X_{t}-\left(\xi+\sigma_{0} b_{T}^{H, \gamma}\right)\right) \stackrel{\mathrm{D}}{\rightarrow} \Lambda .
$$

Although Example 4.1 shows that $H$-proper triples may exist for certain models for all $H \in(0,1)$, they indeed only exist for Vasicek models (see [5, Remark 3.3(vii)]). When considering more general models we restrict ourselves to a choice of $H \in\left(\frac{1}{2}, 1\right)$, which is uncritical for most models.

Formulae (4.1) and (4.2) provide us with two different representations for $f^{-1}$; the first is based on the ratio $\mu / \sigma$ and the second on the integral representation (4.2). The results of Section 3 and asymptotic inversion rules yield different characterizations of the maximum domain of attraction.

We start with the maximum domain of attraction of the Gumbel distribution. The proof of Theorem 4.1 can be found in Appendix C.1. The equivalence of the conditions (i), (ii), and (iii) (of part (b)) is a direct consequence of (4.1) and (4.2).

Theorem 4.1. Let $H \in\left(\frac{1}{2}, 1\right)$. Suppose $(I, \mu, \sigma)$ to be $H$-proper, with friction coefficient $\gamma$, SST $f$, and centre $\xi$. Let $\psi$ be the absolutely continuous extension of $\mu / \sigma$ to I. The following assertions are equivalent.

(a) $X^{H, \gamma, f} \in \operatorname{MDA}(\Lambda)$. 
(b) There exist a $z_{0} \in I$ and a function $g:\left(z_{0}, r\right) \rightarrow \mathbb{R}^{+}$such that, for all $x \in \mathbb{R}$, there exists a $z_{1} \in\left(z_{0}, r\right)$ satisfying $z+x g(z) \in I$ for all $z \in\left(z_{1}, r\right)$, and one of the following equivalent conditions holds for all $x \in \mathbb{R}$ :

(i) $\lim _{z \uparrow r} f^{-1}(z)\left(f^{-1}(z+x g(z))-f^{-1}(z)\right)=x$,

(ii) $\lim _{z \uparrow r} \gamma^{-2} \psi(z)(\psi(z+x g(z))-\psi(z))=x$,

(iii) $\lim _{z \uparrow r} \int_{\xi}^{z} \frac{\mathrm{d} w}{\sigma(w)} \int_{z}^{z+x g(z)} \frac{\mathrm{d} w}{\sigma(w)}=x$.

In the case $r=\infty$, the proof of the following corollary illustrates a possible construction of $g$ as in Theorem 4.1(b). Analogous results hold for $r<\infty$.

Corollary 4.1. Let $H \in\left(\frac{1}{2}, 1\right)$. Suppose $(I, \mu, \sigma)$ to be $H$-proper, with friction coefficient $\gamma, \operatorname{SST} f$, and centre $\xi$. Suppose that $r=\infty$ and that there exists a $z_{0} \in I$ such that $\ell:\left(z_{0}, \infty\right) \rightarrow \mathbb{R}^{+}$is a slowly varying function. Then

(a) if there exists a $p<1$ such that $\sigma(z)=z^{p} \ell(z)$ for all $z>z_{0}>\max \{0, \xi\}$, we have $X^{H, \gamma, f} \in \operatorname{MDA}(\Lambda) ;$ and

(b) if there exists a $q<\frac{1}{2}$ such that $\sigma(z)=z(\log z)^{q} \ell(\log z)$ for all $z>z_{0}>\max \left\{1, \mathrm{e}^{\xi}\right\}$, we have $X^{H, \gamma, f} \in \operatorname{MDA}(\Lambda)$.

Proof. In both cases, we check condition (iii) of Theorem 4.1(b).

(a) Define $g:\left(z_{0}, \infty\right) \rightarrow \mathbb{R}^{+}$by $g(z)=\sigma(z) / \int_{\xi}^{z} \sigma^{-1}(w) \mathrm{d} w$. Karamata's theorem [3, Theorem 1.6.1] implies that

$$
\lim _{z \rightarrow \infty} \frac{g(z)}{z}=(1-p) \lim _{z \rightarrow \infty} \ell^{2}(z) z^{2 p-2}=0 .
$$

Thus, for all $x \in \mathbb{R}$, we can find a $z_{1}>z_{0}$ such that $z+g(z) x \subseteq\left(z_{0}, \infty\right)$ for all $z>z_{1}$. In particular, as $\ell$ is strictly positive and $\sigma: I \rightarrow \mathbb{R}($ and $1 / \sigma)$ are continuous on $\left(z_{1}, \infty\right)$. Consequently, for $z>z_{1}$, the mean value theorem provides a $\theta(z) \in[0,1]$ such that

$$
\int_{z}^{z+x g(z)} \frac{\mathrm{d} w}{\sigma(w)}=\frac{x g(z)}{\sigma(z+\theta(z) x g(z))} .
$$

On the other hand, by definition,

$$
\int_{\xi}^{z} \frac{\mathrm{d} w}{\sigma(w)} \int_{z}^{z+x g(z)} \frac{\mathrm{d} w}{\sigma(w)}=\frac{x \sigma(z)}{\sigma\left(z\left(1+\theta(z) x g(z) z^{-1}\right)\right)} .
$$

The right-hand side tends to $x$ for $z \rightarrow \infty$, as $g(z) / z \rightarrow 0$, and convergence in regular variation is locally uniform on $(0, \infty)$ (see [3, Theorem 1.5.2]).

(b) Define $g:\left(z_{0}, \infty\right) \rightarrow \mathbb{R}^{+}$by $g(z)=\sigma(z) / \int_{\xi}^{z} \sigma^{-1}(w) \mathrm{d} w$, as in the proof of part (a). Substituting $y=\log w$ yields

$$
\int_{z_{0}}^{z} \sigma^{-1}(w) \mathrm{d} w=\int_{\log z_{0}}^{\log z} \frac{1}{y^{q} \ell(y)} \mathrm{d} y .
$$


Karamata's theorem [3, Theorem 1.6.1] implies that

$$
\lim _{z \rightarrow \infty} \frac{g(z)}{z}=(1-q) \lim _{z \rightarrow \infty} \ell(\log z)^{2}(\log z)^{2 q-1}=0 .
$$

Thus, for all $x \in \mathbb{R}$, we can find a $z_{1}>z_{0}$ such that $z+g(z) x \subseteq\left(z_{0}, \infty\right)$ for all $z>z_{1}$. The remaining part of the proof follows along the same lines as does that of part (a).

Theorem 3.2 yields a characterization of $\operatorname{MDA}\left(\Phi_{\alpha}\right)$ in the following theorem; see Appendix C.2 for a proof. The equivalence of the representations (i), (ii), and (iii) (of part (b)) is a direct consequence of (4.1) and (4.2).

Theorem 4.2. Let $H \in\left(\frac{1}{2}, 1\right)$ and let $(I, \mu, \sigma)$ be $H$-proper, with friction coefficient $\gamma, S S T f$, and centre $\xi$. Let $\psi$ be the absolutely continuous extension of $\mu / \sigma$ to $I$. The following assertions are equivalent.

(a) There exists an $\alpha>0$ such that $X \in \operatorname{MDA}\left(\Phi_{\alpha}\right)$.

(b) We have $r=\infty$ and there exist a $\kappa>0$ and a function $\tilde{h}:(\max \{1, l\}, \infty) \rightarrow \mathbb{R}$ such that both

$$
\lim _{z \rightarrow \infty}(\log z)^{1 / 2}(\tilde{h}(x z)-\tilde{h}(z))=0 \text { for all } x>0
$$

and one of the following equivalent representations holds for all $z>\max \{1, l\}$ :

(i) $f^{-1}(z)=(2 / \kappa)^{1 / 2}(\log z)^{1 / 2}+\tilde{h}(z)$,

(ii) $\psi(z)=-\gamma\left((2 / \kappa)^{1 / 2}(\log z)^{1 / 2}+\tilde{h}(z)\right)$,

(iii) $\int_{\xi}^{z} \frac{\mathrm{d} w}{\sigma(w)}=\left(\frac{2}{\kappa}\right)^{1 / 2}(\log z)^{1 / 2}+\tilde{h}(z)$.

If either condition (a) or condition (b) is satisfied then $\alpha=\delta^{H, \gamma} / \kappa$, where $\delta^{H, \gamma}$ is the quantity in Remark 2.1(b).

As an application of Corollary 4.1 and Theorem 4.2, we present a family of models that, depending on the choice of parameters, belong to either $\operatorname{MDA}(\Lambda)$ or $\operatorname{MDA}\left(\Phi_{\alpha}\right)$.

Example 4.2. Let $H \in\left(\frac{1}{2}, 1\right), q \in((1-H), 1), \sigma_{0}>0, a<0$, and $b \geq 0$. Calculations similar to those of $[5$, Section 5] show that $(I, \mu, \sigma)$ with

$$
I=\mathbb{R}^{+}, \quad \mu(z)=a z \log z+b z|\log z|^{q}, \quad \sigma(z)=\sigma_{0} z|\log z|^{q}
$$

is $H$-proper. Furthermore, (4.1) shows that $\gamma=(1-q)|a|$. We obtain two cases, as follows. For $q=\frac{1}{2}$, we observe that

$$
\psi(z)=\frac{a}{\sigma_{0}}(\log z)^{1 / 2}+\frac{b}{\sigma_{0}}, \quad z>1 .
$$

Set $\kappa=\frac{1}{2} \sigma_{0}^{2}$ and $\tilde{h}(z) \equiv b / \sigma_{0}$. Theorem 4.2(b) applies to $\psi$; thus, $X^{H, \gamma, f} \in \operatorname{MDA}\left(\Phi_{\alpha}\right)$ for $\alpha=2 \delta^{H, \gamma} / \sigma_{0}^{2}$. For $q<\frac{1}{2}$, Corollary 4.1(b) implies that $X^{H, \gamma, f} \in \operatorname{MDA}(\Lambda)$.

Calculations show that the SST $f$ can be explicitly written as

$$
f(z)=\exp \left(\operatorname{sgn}\left(\sigma_{0}(1-q) z-\frac{b}{a}\right)\left|\sigma_{0}(1-q) z-\frac{b}{a}\right|^{1 /(1-q)}\right), \quad z \in \mathbb{R} .
$$


Hence, we could also have made our argument using the theory given in Section 3 . In this case, Example 3.1 shows that $X^{H, \gamma, f} \notin \operatorname{MDA}(G)$ for any extreme-value distribution $G$ and any $q \in\left(\frac{1}{2}, 1\right)$.

For completeness, we conclude the section with the corresponding results for $\operatorname{MDA}\left(\Psi_{\alpha}\right)$. The following theorem is based on Theorem 3.3; its proof can be found in Appendix C.3.

Theorem 4.3. Let $H \in\left(\frac{1}{2}, 1\right)$ and let $(I, \mu, \sigma)$ be $H$-proper, with friction coefficient $\gamma, S S T f$, and centre $\xi$. Let $\psi$ be the absolutely continuous extension of $\mu / \sigma$ to $I$. The following assertions are equivalent.

(a) There exists an $\alpha>0$ such that $X \in \operatorname{MDA}\left(\Psi_{\alpha}\right)$.

(b) We have $r<\infty$ and there exist a $\kappa>0$ and a function $\bar{h}:(0, r-l) \rightarrow \mathbb{R}$ such that both

$$
\lim _{z \downarrow 0}|\log z|^{1 / 2}(\bar{h}(x z)-\bar{h}(z))=0 \quad \text { for all } x>0
$$

and one of the following equivalent representations holds for all $z, 0<z<\min \{1$, $r-l\}$ :

$$
\begin{aligned}
& \text { (i) } f^{-1}(r-z)=(2 / \kappa)^{1 / 2}|\log z|^{1 / 2}+\bar{h}(z), \\
& \text { (ii) } \psi(r-z)=-\gamma(2 / \kappa)^{1 / 2}|\log z|^{1 / 2}+\bar{h}(z), \\
& \text { (iii) } \int_{\xi}^{r-z} \frac{\mathrm{d} w}{\sigma(w)}=\left(\frac{2}{\kappa}\right)^{1 / 2}|\log z|^{1 / 2}+\bar{h}(z) .
\end{aligned}
$$

If either condition (a) or condition (b) is satisfied then $\alpha=\delta^{H, \gamma} / \kappa$, where $\delta^{H, \gamma}$ is the quantity in Remark 2.1(b).

\section{Appendix A. Proof of Lemma 2.1}

It remains to show parts (b) and (d) of Lemma 2.1.

(b) By the self-similarity of fractional Brownian motion, we obtain

$$
\begin{aligned}
\rho_{H, \gamma, \sigma}(h) & =\sigma^{2} \mathrm{E}\left(\int_{-\infty}^{0} \mathrm{e}^{\gamma s} \mathrm{~d} B_{s}^{H} \int_{-\infty}^{h} \mathrm{e}^{-\gamma(h-s)} \mathrm{d} B_{s}^{H}\right) \\
& =\sigma^{2} \mathrm{e}^{-\gamma h} \mathrm{E}\left(\int_{-\infty}^{0} \mathrm{e}^{s} \mathrm{~d} B_{s / \gamma}^{H} \int_{-\infty}^{\gamma h} \mathrm{e}^{s} \mathrm{~d} B_{s / \gamma}^{H}\right) \\
& =\frac{\sigma^{2}}{\gamma^{2 H}} \rho_{H}(\gamma h)
\end{aligned}
$$

for $h \in \mathbb{R}$.

(d) The closed formula stated for $H=\frac{1}{2}$ is well known (see, e.g. [7]). Thus, by parts (a) and (b), it suffices to investigate the case with $\gamma=\sigma=1, H \neq \frac{1}{2}$, and $h \downarrow 0$.

By partial integration applied to (1.3), we observe that

$$
\int_{-\infty}^{t} \mathrm{e}^{-(t-s)} \mathrm{d} B_{s}^{H}=B_{t}^{H}-\int_{-\infty}^{t} \mathrm{e}^{-(t-s)} B_{s}^{H} \mathrm{~d} s, \quad t \in \mathbb{R},
$$


where the integral on the right-hand side is interpreted as a Lebesgue integral (cf. [5, Proposition 2.3]).

By (A.1) and Fubini's theorem, we have

$$
\begin{aligned}
\rho_{H}(0) & =\int_{-\infty}^{0} \int_{-\infty}^{0} \mathrm{e}^{s_{1}+s_{2}} \mathrm{E}\left(B_{s_{1}}^{H} B_{s_{2}}^{H}\right) \mathrm{d} s_{1} \mathrm{~d} s_{2} \\
& =\frac{1}{2} \int_{-\infty}^{0} \int_{-\infty}^{0} \mathrm{e}^{s_{1}+s_{2}}\left(\left|s_{1}\right|^{2 H}+\left|s_{2}\right|^{2 H}-\left|s_{1}-s_{2}\right|^{2 H}\right) \mathrm{d} s_{1} \mathrm{~d} s_{2} \\
& =\Gamma(2 H+1)-\frac{1}{2} \int_{0}^{\infty} \int_{0}^{\infty} \mathrm{e}^{-\left(s_{1}+s_{2}\right)}\left|s_{1}-s_{2}\right|^{2 H} \mathrm{~d} s_{1} \mathrm{~d} s_{2} .
\end{aligned}
$$

The integral on the final line can be interpreted as a multiple of the expectation $\mathrm{E}\left|S_{1}-S_{2}\right|^{2 H}$, where $S_{1}$ and $S_{2}$ are independent standard exponential random variables. As $S_{1}-S_{2}$ is a two-sided exponential random variable, we obtain

$$
\int_{0}^{\infty} \int_{0}^{\infty} \mathrm{e}^{-\left(s_{1}+s_{2}\right)}\left|s_{1}-s_{2}\right|^{2 H} \mathrm{~d} s_{1} \mathrm{~d} s_{2}=\frac{1}{2} \int_{-\infty}^{\infty} \mathrm{e}^{-|s|}|s|^{2 H} \mathrm{~d} s=\Gamma(2 H+1) .
$$

Hence,

$$
\rho_{H}(0)=\frac{1}{2} \Gamma(2 H+1)
$$

Now let $h \geq 0$ and set

$$
\begin{aligned}
& \phi_{H}(h)=\Gamma(2 H+1)-\int_{0}^{\infty} \mathrm{e}^{-s}(h+s)^{2 H} \mathrm{~d} s \\
& \psi_{H}(h)=\frac{1}{2}\left(\Gamma(2 H+1) \int_{0}^{h} \mathrm{e}^{s} \mathrm{~d} s+\int_{0}^{h} s^{2 H} \mathrm{e}^{s} \mathrm{~d} s-\int_{0}^{h} \mathrm{e}^{s_{1}} \int_{0}^{\infty} \mathrm{e}^{-s_{2}}\left(s_{1}+s_{2}\right)^{2 H} \mathrm{~d} s_{2} \mathrm{~d} s_{1}\right) .
\end{aligned}
$$

By Fubini's theorem and (1.1), we have

$$
\mathrm{E}\left(B_{h}^{H} \int_{-\infty}^{0} \mathrm{e}^{s} B_{s}^{H} \mathrm{~d} s\right)=\frac{1}{2} \int_{-\infty}^{0} \mathrm{e}^{s}\left(h^{2 H}-s^{2 H}-(h-s)^{2 H}\right) \mathrm{d} s=\frac{1}{2}\left(h^{2 H}+\phi_{H}(h)\right)
$$

and, similarly, $\psi_{H}(h)=\mathrm{E}\left(\int_{-\infty}^{0} \mathrm{e}^{s} B_{s}^{H} \mathrm{~d} s \int_{0}^{h} \mathrm{e}^{s} B_{s}^{H} \mathrm{~d} s\right)$.

By (A.1),

$$
\begin{aligned}
\rho_{H}(h)-\rho_{H}(0)= & -\mathrm{E}\left(\int_{-\infty}^{0} \mathrm{e}^{s} B_{s}^{H} \mathrm{~d} s\left(B_{h}^{H}-\mathrm{e}^{-h} \int_{-\infty}^{h} \mathrm{e}^{s} B_{s}^{H} \mathrm{~d} s+\int_{-\infty}^{0} \mathrm{e}^{s} B_{s}^{H} \mathrm{~d} s\right)\right) \\
= & -\mathrm{E}\left(B_{h}^{H} \int_{-\infty}^{0} \mathrm{e}^{s} B_{s}^{H} \mathrm{~d} s+\left(\mathrm{e}^{-h}-1\right) \mathrm{E} \int_{-\infty}^{h} \mathrm{e}^{s} B_{s}^{H} \mathrm{~d} s \int_{-\infty}^{0} \mathrm{e}^{s} B_{s}^{H} \mathrm{~d} s\right) \\
& +\mathrm{E}\left(\int_{-\infty}^{0} \mathrm{e}^{s} B_{s}^{H} \mathrm{~d} s \int_{0}^{h} \mathrm{e}^{s} B_{s}^{H} \mathrm{~d} s\right) \\
= & -\frac{1}{2}\left(h^{2 H}+\phi_{H}(h)\right)+\left(\mathrm{e}^{-h}-1\right)\left(\frac{1}{2} \Gamma(2 H+1)+\psi_{H}(h)\right)+\psi_{H}(h) .
\end{aligned}
$$

For $H<\frac{1}{2}$, we can differentiate both $\phi_{H}(h)$ and $\psi_{H}(h)$ under the integral sign, by dominated convergence. We obtain

$$
\begin{aligned}
& \phi_{H}(h)=\phi_{H}(0)+\phi_{H}^{\prime}(0+) h+o(h)=-\Gamma(2 H+1) h+o(h), \\
& \psi_{H}(h)=\psi_{H}(0)+\psi_{H}^{\prime}(0+) h+o(h)=o(h) .
\end{aligned}
$$


Equation (A.3) yields

$$
\rho_{H}(h)-\rho_{H}(0)=-\frac{1}{2} h^{2 H}+\frac{1}{2} \Gamma(2 H+1) h-\frac{1}{2} \Gamma(2 H+1) h+o(h)=-\frac{1}{2} h^{2 H}+o(h) .
$$

By (A.2) and part (b), we find that

$$
\rho_{H, \gamma, \sigma}(h)=\frac{\sigma^{2}}{\gamma^{2 H}}\left(\rho_{H}(0)+\rho_{H}(\gamma h)-\rho_{H}(0)\right)=\frac{\Gamma(2 H+1)}{2} \frac{\sigma^{2}}{\gamma^{2 H}}-\frac{1}{2} \sigma^{2} h^{2 H}+o(h) .
$$

For $H>\frac{1}{2}$, both $\phi_{H}$ and $\psi_{H}$ are twice differentiable under the integral sign, i.e.

$$
\begin{aligned}
& \phi_{H}(h)=-\Gamma(2 H+1) h-\frac{1}{2} \Gamma(2 H+1) h^{2}+o\left(h^{2}\right), \\
& \psi_{H}(h)=-\frac{1}{4} \Gamma(2 H+1) h^{2}+o\left(h^{2}\right) ;
\end{aligned}
$$

thus, as $\mathrm{e}^{-h}-1=-h+\frac{1}{2} h^{2}+o\left(h^{2}\right),($ A.3) implies that

$$
\rho_{H}(h)-\rho_{H}(0)=-\frac{1}{2} h^{2 H}+\frac{1}{4} \Gamma(2 H+1) h^{2}+o\left(h^{2}\right) .
$$

By the same arguments as above, we have

$$
\rho_{H, \gamma, \sigma}(h)=\frac{\Gamma(2 H+1)}{2} \frac{\sigma^{2}}{\gamma^{2 H}}-\frac{1}{2} \sigma^{2} h^{2 H}+\frac{\Gamma(2 H+1)}{4} \frac{\sigma^{2}}{\gamma^{2 H-2}} h^{2}+o\left(h^{2}\right) .
$$

\section{Appendix B. A general convergence-to-types lemma}

In this section, we state a result that forms the core of Section 3. For a probability distribution function $F: \mathbb{R} \rightarrow[0,1]$, we write

$$
D_{<}(F)=\{x \in \mathbb{R}: \text { for all } \varepsilon>0, F(x-\varepsilon)<F(x)<F(x+\varepsilon)\} .
$$

Set $x_{\mathrm{L}}:=-\infty$ if $F(x)>0$ for all $x \in \mathbb{R}$; otherwise, set $x_{\mathrm{L}}=\sup \{x \in \mathbb{R}: F(x)=0\}$. Set $x_{\mathrm{R}}:=\infty$ if $F(x)<1$ for all $x \in \mathbb{R}$; otherwise, set $x_{\mathrm{R}}=\inf \{x \in \mathbb{R}: F(x)=1\}$.

Lemma B.1. Let $F, F_{n}: \mathbb{R} \rightarrow[0,1], n \in \mathbb{N}$, be probability distribution functions on $\mathbb{R}$, with $F$ continuous.

(a) Let $M=\left(x_{\mathrm{L}}, x_{\mathrm{R}}\right)$ and consider a function $g_{n}: M \rightarrow \mathbb{R}, n \in \mathbb{N}$. Let $G_{n}=F_{n} \circ g_{n}: M \rightarrow$ $[0,1]$. If

$$
\lim _{n \rightarrow \infty} g_{n}(x)=x \text { and } \lim _{n \rightarrow \infty} G_{n}(x)=F(x)
$$

for all $x \in M$, then

$$
\lim _{n \rightarrow \infty} F_{n}(x)=F(x) \text { for all } x \in \mathbb{R} .
$$

(b) Let $M=D_{<}(F)$ and consider a function $g_{n}: M \rightarrow \mathbb{R}, n \in \mathbb{N}$. Again, let $G_{n}=F_{n} \circ g_{n}$ : $M \rightarrow[0,1]$. If

$$
\lim _{n \rightarrow \infty} F_{n}(x)=\lim _{n \rightarrow \infty} G_{n}(x)=F(x)
$$

for all $x \in M$, then $g_{n}(x) \rightarrow x$ for all $x \in M$. 
Proof. (a) It suffices to show that $\lim _{n \rightarrow \infty} F_{n}(x)=F(x)$ for all $x \in M$. In contradiction to the hypothesis, suppose that there exist an $x_{0} \in M$ and a $y_{0} \in[0,1]$ and, as $F_{n}(x)$ is bounded, a subsequence $n^{\prime}$ such that

$$
\lim _{n^{\prime} \rightarrow \infty} F_{n^{\prime}}\left(x_{0}\right)=y_{0} \neq F\left(x_{0}\right) .
$$

Without loss of generality, suppose that $n=n^{\prime}$. By Helly's selection theorem, we can find a subsequence $n^{\prime}$ and a nondecreasing, right-continuous function $\tilde{F}: \mathbb{R} \rightarrow[0,1]$ such that $\lim _{n^{\prime} \rightarrow \infty} F_{n^{\prime}}(x)=\tilde{F}(x)$ for all continuity points $x$ of $\tilde{F}$. Let $C(\tilde{F})$ be the set of such continuity points and let $x \in C(\tilde{F}) \cap\left(x_{\mathrm{L}}, x_{\mathrm{R}}\right)$. Then, for all $x^{\prime} \in\left(x, x_{\mathrm{R}}\right) \cap C(\tilde{F})$, we have

$$
F(x)=\lim _{n^{\prime} \rightarrow \infty} G_{n^{\prime}}(x)=\lim _{n^{\prime} \rightarrow \infty} F_{n^{\prime}}\left(g_{n^{\prime}}(x)\right) \leq \lim _{n^{\prime} \rightarrow \infty} F_{n^{\prime}}\left(x^{\prime}\right)=\tilde{F}\left(x^{\prime}\right)
$$

and, hence, $F(x) \leq \lim _{x^{\prime} \downarrow x, x^{\prime} \in C(\tilde{F})} \tilde{F}\left(x^{\prime}\right)=\tilde{F}(x)$. Analogously, for all $x^{\prime} \in\left(x_{\mathrm{L}}, x\right) \cap C(\tilde{F})$, we have

$$
\tilde{F}\left(x^{\prime}\right)=\lim _{n^{\prime} \rightarrow \infty} F_{n^{\prime}}\left(x^{\prime}\right) \leq \lim _{n^{\prime} \rightarrow \infty} F_{n^{\prime}}\left(g_{n^{\prime}}(x)\right)=\lim _{n^{\prime} \rightarrow \infty} G_{n^{\prime}}(x)=F(x) .
$$

Hence, $\tilde{F}(x)=\lim _{x^{\prime} \uparrow x, x^{\prime} \in C(\tilde{F})} \tilde{F}\left(x^{\prime}\right) \leq F(x)$ and, so, $\tilde{F}(x)=F(x)$ for all $x \in\left(x_{\mathrm{L}}, x_{\mathrm{R}}\right) \cap$ $C(\tilde{F})$. As $C(\tilde{F})$ is dense in $\left(x_{\mathrm{L}}, x_{\mathrm{R}}\right)$ and $F$ is continuous, we find that $x_{0} \in\left(x_{\mathrm{L}}, x_{\mathrm{R}}\right) \subseteq C(\tilde{F})$, contradicting (B.1).

(b) Suppose that the contrary is true. Then there exist an $x \in D_{<}(F)$ and a subsequence $n^{\prime}$ such that $g_{n^{\prime}}(x) \rightarrow y \in \overline{\mathbb{R}}=\mathbb{R} \cup\{\infty\}$, where $y \neq x$. Without loss of generality, suppose that $y \in[-\infty, x)$. As $F$ is continuous, uniform convergence of $F_{n} \rightarrow F$ holds. If we set $F(y)=0$ whenever $y=-\infty$, then

$$
F(y)=\lim _{n^{\prime} \rightarrow \infty} F_{n^{\prime}}\left(g_{n^{\prime}}(x)\right)=\lim _{n^{\prime} \rightarrow \infty} G_{n^{\prime}}(x)=F(x),
$$

contradicting our assumption that $x \in D_{<}(F)$.

\section{Appendix C. Results on asymptotic inversion}

\section{C.1. Proof of Theorem 4.1}

Theorem 4.1 is a consequence of Theorem 3.1 and the following lemma.

Lemma C.1. Let $f$ be an SST with state space $I=(l, r)$. The following assertions are equivalent.

(a) The SST $f$ satisfies (3.1).

(b) There exist $a z_{0} \in I$ and a function $g:\left(z_{0}, r\right) \rightarrow \mathbb{R}^{+}$satisfying the following properties:

(i) for all $x \in \mathbb{R}$, there exists a $z_{1} \in\left(z_{0}, r\right)$ with $z+x g(z) \in I$ for all $z \in\left(z_{1}, r\right)$;

(ii) $\lim _{z \uparrow r} f^{-1}(z)\left(f^{-1}(z+x g(z))-f^{-1}(z)\right)=x$ for all $x \in \mathbb{R}$.

Proof. We first prove that part (a) implies part (b). The SST $f$ has representation $f(z)=$ $v \circ h(z)$ for all $z>0$, where $v$ is an arbitrary function and $h(z)=\mathrm{e}^{z^{2} / 2}$. By combining Exercises 0.4.3.7 and 0.4.3.8 of [13], we can find a function $a:(1, \infty) \rightarrow \mathbb{R}^{+}$such that $\lim _{z \rightarrow \infty}[v(z x)-v(z)] / a(z)=\log x$ for all $x>0$. As both $f$ and $h$ are strictly increasing, so 
too is $v$; moreover, $\lim _{z \uparrow r} v^{-1}(z)=\infty$. Let $z_{0}=f(0)$. From [13, Proposition 0.9(b)], we can find a function $g:\left(z_{0}, r\right) \rightarrow \mathbb{R}^{+}$, satisfying part (b)(i), such that

$$
\lim _{z \uparrow r} \frac{v^{-1}(z+x g(z))}{v^{-1}(z)}=\mathrm{e}^{x}
$$

for all $x \in \mathbb{R}$. Part (b)(ii) follows from the fact that, for $x \in \mathbb{R}$,

$$
\begin{aligned}
& \lim _{z \uparrow r} f^{-1}(z)\left(f^{-1}(z+x g(z))-f^{-1}(z)\right) \\
& \quad=\lim _{z \uparrow r} 2\left[\log v^{-1}(z)\right]\left[\left(1+\frac{\log \left(v^{-1}(z+x g(z)) / v^{-1}(z)\right)}{\log v^{-1}(z)}\right)^{1 / 2}-1\right] .
\end{aligned}
$$

A Taylor expansion of $(1+z)^{1 / 2}$ now yields the assertion.

We now prove that part (b) implies part (a). Observe that, for all $x \in \mathbb{R}$,

$$
\lim _{z \uparrow r}\left[f^{-1}(z)\right]^{2}\left[\frac{f^{-1}(z+x g(z))}{f^{-1}(z)}-1\right]=x .
$$

Consequently, $\lim _{z \uparrow r} f^{-1}(z+x g(z)) / f^{-1}(z)=1$, as $\lim _{z \uparrow r} f^{-1}(z)=\infty$. Now define $u(z)=\exp \left(\frac{1}{2}\left(f^{-1}(z)\right)^{2}\right), z \in I$. Then $u$ is strictly increasing on $(f(0), r)$ and provides a mapping from $(f(0), r)$ onto $(u(f(0)), \infty)$. For all $x \in \mathbb{R}$, we find that

$$
\begin{aligned}
& \lim _{z \uparrow r} \frac{u(z+g(z) x)}{u(z)} \\
& \quad=\lim _{z \uparrow r} \exp \left(\frac{1}{2} \frac{f^{-1}(z+x g(z))+f^{-1}(z)}{f^{-1}(z)} f^{-1}(z)\left(f^{-1}(z+x g(z))-f^{-1}(z)\right)\right)=\mathrm{e}^{x} .
\end{aligned}
$$

Proposition 0.9(a) of [13] applies to $u$. There exist a $z_{1}>u(f(0))$ and a function $a:\left(z_{1}, \infty\right) \rightarrow \mathbb{R}^{+}$such that $\lim _{z \rightarrow \infty}\left(u^{-1}(z x)-u^{-1}(z)\right) / a(z)=\log x$ for all $x>0$. By monotonicity, the convergence holds locally uniformly in $x$ on $\mathbb{R}^{+}$. In particular, for all $x \in \mathbb{R}$, we have

$$
\begin{aligned}
& \lim _{z \rightarrow \infty} \frac{f\left(z+x z^{-1}\right)-f(z)}{a\left(\mathrm{e}^{z^{2} / 2}\right)} \\
& \quad=\lim _{z \rightarrow \infty} \frac{u^{-1}\left(\mathrm{e}^{z^{2} / 2} \exp \left(x+x^{2} /\left(2 z^{2}\right)\right)\right)-u^{-1}\left(\mathrm{e}^{z^{2} / 2}\right)}{a\left(\mathrm{e}^{z^{2} / 2}\right)}=x .
\end{aligned}
$$

Therefore, for all $x \in \mathbb{R}$, we have

$$
\lim _{z \rightarrow \infty} \frac{f\left(z+x z^{-1}\right)-f(z)}{f\left(z+z^{-1}\right)-f(z)}=\lim _{z \rightarrow \infty} \frac{f\left(z+x z^{-1}\right)-f(z)}{a\left(\mathrm{e}^{z^{2} / 2}\right)} \frac{a\left(\mathrm{e}^{z^{2} / 2}\right)}{f\left(z+z^{-1}\right)-f(z)}=x .
$$

\section{C.2. Proof of Theorem 4.2}

We prepare for the result with a technical lemma.

Lemma C.2. If $h:\left(x_{0}, \infty\right) \rightarrow \mathbb{R}$ with $\lim _{z \rightarrow \infty} z^{\alpha}\left(h\left(z+x z^{-\beta}\right)-h(z)\right)=0$ locally uniformly in $x \in \mathbb{R}$ for some $x_{0} \in \mathbb{R}, \alpha \in[0,1)$, and $\beta \geq 0$, then $\lim _{z \rightarrow \infty} z^{\alpha-1-\beta} h(z)=0$. 
Proof. We use the convention $\sum_{k}^{l}=0$ for $l<k$. Let $\varepsilon>0$ and define a sequence $\left(z_{n}\right)$ as follows. Choose $z_{0}>\max \left\{1, x_{0}\right\}$ such that, for all $z \geq z_{0}$ and $x \in[0,1]$, we have

$$
\left|h\left(z+x z^{-\beta}\right)-h(z)\right|<\varepsilon\left(z+z^{-\beta}\right)^{-\alpha} .
$$

For $n \geq 1$, set $z_{n}=z_{n-1}+z_{n-1}^{-\beta}$. Observe that $z_{n}=z_{0}+\sum_{l=0}^{n-1} z_{l}^{-\beta} \geq n z_{n}^{-\beta}$. Thus, $z_{n} \geq n^{\overline{1 /}(1+\beta)}$. In particular, $z_{n} \rightarrow \infty$ and $\left|h\left(z_{n+1}\right)-h\left(z_{n}\right)\right|<\varepsilon(n+1)^{-\alpha /(1+\beta)}$ for all $n \geq 0$.

Let $z \geq z_{0}$ be arbitrary. Set $n_{1}=\max \left\{n: z_{n} \leq z\right\}$; then clearly $n_{1} \leq z^{1+\beta}$. By this choice of $n_{1}$, we have $z \in\left[z_{n_{1}}, z_{n_{1}}+z_{n_{1}}^{-\beta}\right.$ ) and, hence, $\left|h(z)-h\left(z_{n_{1}}\right)\right|<\varepsilon$. Finally, summing and subtracting terms gives

$$
|h(z)| \leq \varepsilon+\left|h\left(z_{0}\right)\right|+\varepsilon \sum_{k=0}^{n_{1}-1}(1+k)^{-\alpha /(1+\beta)} \leq \varepsilon+\left|h\left(z_{0}\right)\right|+\varepsilon\left(1+\frac{1+\beta}{1-\alpha+\beta} z^{1-\alpha+\beta}\right) .
$$

Thus, $\lim \sup _{z \rightarrow \infty} z^{\alpha-1-\beta}|h(z)| \leq \varepsilon$.

Corollary C.1. (a) If $x_{0} \in \mathbb{R}$ and $h:\left(x_{0}, \infty\right) \rightarrow \mathbb{R}$ with

$$
\lim _{z \rightarrow \infty}\left(h\left(z+x z^{-1}\right)-h(z)\right)=0
$$

locally uniformly in $x \in \mathbb{R}$, then $\lim _{z \rightarrow \infty} z^{-2} h(z)=0$.

(b) If $x_{0} \in \mathbb{R}$ and $h:\left(x_{0}, \infty\right) \rightarrow \mathbb{R}$ with

$$
\lim _{z \rightarrow \infty}(\log z)^{1 / 2}(h(z x)-h(z))=0
$$

locally uniformly in $x \in \mathbb{R}^{+}$, then $\lim _{z \rightarrow \infty}(\log z)^{-1 / 2} h(z)=0$.

Proof. For the choice of $\beta=1$ and $\alpha=0$, Lemma C.2 implies part (a). To show part (b), set $g=h \circ$ exp. Then $\lim _{z \rightarrow \infty} z^{1 / 2}(g(z+x)-g(z))=0$ locally uniformly in $x \in \mathbb{R}$ and Lemma C.2 yields $\lim _{z \rightarrow \infty} z^{-1 / 2} g(z)=0$; equivalently, $\lim _{z \rightarrow \infty}(\log z)^{-1 / 2} h(z)=0$.

Proof of Theorem 4.2. We first prove that part (a) implies part (b). Observe that, for all $x \in \mathbb{R}$,

$$
\lim _{z \rightarrow \infty} \frac{f(z+x / z)}{f(z)}=\mathrm{e}^{\kappa x}
$$

This convergence is strengthened to locally uniform convergence by [3, Proposition 3.10.2]. By Theorem 3.2, having $X^{H, \gamma, f} \in \operatorname{MDA}\left(\Phi_{\alpha}\right)$ for $\alpha>0$ is equivalent to the existence of a $z_{0} \in \mathbb{R}$, a $\kappa>0$, and a function $h:\left(z_{0}, \infty\right) \rightarrow \mathbb{R}$ satisfying (3.7) such that both $f(z)>0$ and $\log f(z)=\frac{1}{2} \kappa z^{2}+h(z)$ hold for all $z>z_{0}$. Consequently, $h(z+x / z)-h(z) \rightarrow 0$ as $z \rightarrow \infty$ locally uniformly in $x \in \mathbb{R}$; thus, $z^{-2} h(z) \rightarrow 0$ by Corollary C.1(a). In particular, $f^{-1}(z) \sim(2 / \kappa)^{1 / 2}(\log z)^{1 / 2}$ for $z \rightarrow \infty$.

By [3, Theorem 3.10.4], (C.1) implies that

$$
\lim _{z \rightarrow \infty} f^{-1}(z)\left(f^{-1}(z x)-f^{-1}(z)\right)=\kappa^{-1} \log x
$$

for all $x>0$ or, equivalently, that

$$
\lim _{z \rightarrow \infty}(\log z)^{1 / 2}\left(f^{-1}(z x)-f^{-1}(z)\right) \rightarrow(2 \kappa)^{-1 / 2} \log x
$$


for all $x>0$. Finally, set $\tilde{h}(z)=f^{-1}(z)-(2 / \kappa)^{1 / 2}(\log z)^{1 / 2}$ for $z>\max \{1, l\}$. Then $\tilde{h}$ is a function satisfying (4.3).

We now prove that part (b) implies part (a). Observe that

$$
\lim _{z \rightarrow \infty}(\log z)^{1 / 2}\left(f^{-1}(x z)-f^{-1}(z)\right)=(2 \kappa)^{-1 / 2} \log x
$$

for all $x>0$. Now let $x>0$ and $x(z) \rightarrow x$ for $z \rightarrow \infty$. By monotonicity, for all $0<\varepsilon<x$, we have

$$
\begin{aligned}
(2 \kappa)^{-1 / 2} \log (x-\varepsilon) & \leq \liminf _{z \rightarrow \infty}(\log z)^{1 / 2}\left(f^{-1}(x z)-f^{-1}(z)\right) \\
& \leq \limsup _{z \rightarrow \infty}(\log z)^{1 / 2}\left(f^{-1}(x z)-f^{-1}(z)\right) \\
& \leq(2 \kappa)^{-1 / 2} \log (x+\varepsilon)
\end{aligned}
$$

Consequently,

$$
\lim _{z \rightarrow \infty}(\log z)^{1 / 2}\left(f^{-1}(x z)-f^{-1}(z)\right)=(2 \kappa)^{-1 / 2} \log x
$$

holds locally uniformly in $x>0$. This implies that

$$
\lim _{z \rightarrow \infty}(\log z)^{1 / 2}(\tilde{h}(x z)-\tilde{h}(z))=0
$$

uniformly in $x>0$. Corollary C.1(b) implies that $f^{-1}(z) \sim(2 / \kappa)^{1 / 2}(\log z)^{1 / 2}$; thus, for all $x>0$, we have

$$
\lim _{z \rightarrow \infty} f^{-1}(z)\left(f^{-1}(x z)-f^{-1}(z)\right)=\kappa^{-1} \log x .
$$

By [3, Theorem 3.10.4], (C.2) implies that $\lim _{z \rightarrow \infty} f(z+x / z) / f(z)=\mathrm{e}^{\kappa z}$. If we now set $h(z)=\log f(z)-\frac{1}{2} \kappa z^{2}$ for $z \in \mathbb{R}$, with $f(z)>0$, then $h$ extends to a function satisfying (3.7).

\section{C.3. Proof of Theorem 4.3}

To show the equivalence of parts (a) and (b), set $\tilde{f}(z)=1 /(r-f(z))$. Then $\tilde{f}$ is an SST with state space $J=\left((r-l)^{-1}, \infty\right)$. By Theorem 3.3, having $X^{H, \gamma, f} \in \operatorname{MDA}\left(\Psi_{\alpha}\right)$ for some $\alpha>0$ is equivalent to the existence of a $z_{0}>\max \{1, l\}$, a $\kappa>0$, and a function $h:\left(z_{0}, r\right) \rightarrow \mathbb{R}$ satisfying (3.6) such that both $\tilde{f}(z)>0$ and $\log \tilde{f}(z)=\frac{1}{2} \kappa z^{2}+h(z)$ hold for all $z>z_{0}$. As in the proof of Theorem 4.2, this holds if and only if there exists a function $\tilde{h}$ satisfying (4.3) such that

$$
\tilde{f}^{-1}(z)=(2 / \kappa)^{1 / 2}(\log z)^{1 / 2}+\tilde{h}(z)
$$

As $\tilde{f}^{-1}(1 / z)=f^{-1}(r-z), 0<z<r-l$, this is equivalent to part (b), where $\bar{h}=\tilde{h}(1 / z)$ satisfies (4.4).

\section{Acknowledgements}

The first author takes pleasure in thanking Sid Resnick for comments on a previous version of the paper while both were visiting the University of Virginia. This led to the present, now shorter, proof of Theorem 4.1. He also thanks Leonard Scott for his kind invitation to Charlottesville and pleasant hospitality during the stay. 


\section{References}

[1] Berman, S. M. (1971). Maxima and high level excursions of stationary Gaussian processes. Trans. Amer. Math. Soc. 160, 65-185.

[2] Berman, S. M. (1992). Sojourns and Extremes of Stochastic Processes. Wadsworth, Belmont, CA.

[3] Bingham, N. H., Goldie, C. M. And Teugels, J. L. (1987). Regular Variation. Cambridge University Press.

[4] Borkovec, M. and Klüppelberg, C. (1998). Extremal behavior of diffusion models in finance. Extremes 1, 47-80. (Correction: 379-380.)

[5] Buchmann, B. ANd KlüPPElberg, C. (2005). Fractional integral equations and state space transforms. To appear in Bernoulli.

[6] Burnecki, K. and Michna, Z. (2002). Simulation of Pickands constants. Prob. Math. Statist. 22, $193-199$.

[7] Cheridito, P., Kawaguchi, H. and Maejima, M. (2003). Fractional Ornstein-Uhlenbeck processes. Electron. J. Prob. 8, 14 pp.

[8] Davis, R. A. (1982). Maximum and minimum of one-dimensional diffusions. Stoch. Process. Appl. 13, 1-9.

[9] Embrechts, P., Klüppelberg, C. And Mikosch, T. (1997). Modelling Extremal Events for Insurance and Finance. Springer, Berlin.

[10] Leadbetter, M. R., Lindgren, G. And Rootzén, H. (1983). Extremes and Related Properties of Random Sequences and Processes. Springer, New York.

[11] PICKands, J., III (1969). Asymptotic properties of the maximum in a stationary Gaussian process. Trans. Amer. Math. Soc. 145, 75-86.

[12] Piterbarg, V. I. (1996). Asymptotic Methods in the Theory of Gaussian Processes and Fields (Trans. Math. Monogr. 148). American Mathematical Society, Providence, RI.

[13] Resnick, S. I. (1987). Extreme Values, Regular Variation, and Point Processes. Springer, New York.

[14] Samorodnitsky, G. and Taqqu, M. S. (1994). Stable Non-Gaussian Random Processes. Chapman and Hall, London. 